\title{
Virtual-Reality-Umgebung für die
}

Visualisierung von Entwicklungszielgrößen

auf Basis des Referenzsystems im Modell der

PGE - Produktgenerationsentwicklung

Felix Pfaff, Simon Rapp, Albert Albers

Die Entwicklung komplexer Systeme mit hohem Innovationspotenzial unter Einhaltung von Kosten- und Risikozielen kann nur durch die systematische Nutzung vorhandener unternehmensinterner und -externer Referenzen gelingen. Für Produktentwickelnde als Entscheidungsträger ist es jedoch schwierig einzuschätzen, welche Auswirkungen die gewählten Referenzen und Variationen auf Entwicklungszielgrößen wie Kosten, Risiko und Innovationspotenzial haben. Das Modell der PGE-Produktgenerationsentwicklung bietet hier das Potenzial, schon früh im Entwicklungsprozess mit Wissen über die Auswirkungen von Referenzen und Variationsarten auf Entwicklungszielgrößen die Entscheidungsgrundlage zu verbessern. Um das Wissen über diese Zusammenhänge dem Entwickelnden zur Verfügung zu stellen, werden in diesem Beitrag zwei Visualisierungsansätze entwickelt. Für einen Konzeptworkshop mit berufserfahrenen Studierenden wird ein diagrammbasierter Ansatz entwickelt, angewandt und evaluiert. Basierend auf den Ergebnissen des Workshops wird eine Virtual-Reality (VR) Visualisierungsumgebung entwickelt und initial in einem Forschungsgespräch validiert. Die VR-Umgebung veranschaulicht dem Nutzer intuitiv und systemspezifisch die Zusammenhänge zwischen dem Referenzsystem und der aktuellen Produktgeneration und deren Auswirkungen auf Entwicklungszielgrößen.

Keywords: Innovationsmanagement, Virtual Reality, Wissensmanagement

\section{Einleitung}

Produktentwickelnde sind in der Verantwortung, unter diversen Randbedingungen aber auch Unsicherheiten Entscheidungen zu treffen und deren Tragweiten auf Entwicklungszielgrößen wie Kosten, Risiko und Innovationspotenzial richtig einzuschätzen. Gerade in frühen Phasen des Produktentwicklungsprozesses ist die Unsicherheit in Projekten hoch, was beim Entscheidungsträger zu Entscheidungsscheu und Verzögerungen im Projekt führen kann. (Cross, 2008, S. 151; Ehrlenspiel \& Meerkamm, 2017, S. 232). 
Aus Sicht der Entwickelnden gilt es, Kosten und Risiko im Produktentwicklungsprozess niedrig zu halten. Erhöhte Kosten und Risiken ergeben sich zum Beispiel durch die Entwicklung neuer Elemente oder die Anpassung von Vorhandenem an einen neuen Kontext. Das Modell der PGE - Produktgenerationsentwicklung beschreibt und erklärt diese Zusammenhänge und bietet das Potenzial, dieses Wissen dem Entwickelnden zugänglich zu machen. (Albers, Rapp, Birk \& Bursac, 2017)

Um dieses Potenzial zu heben, werden in diesem Beitrag zwei Visualisierungsansätze vorgestellt und diskutiert. Für einen Konzeptworkshop mit berufserfahrenen Studierenden wird ein diagrammbasierter Ansatz entwickelt, angewandt und evaluiert. Aufbauend auf den Erkenntnissen aus dieser Vorstudie wird ein VR-Visualisierungsansatz vorgestellt, der dem Entwickelnden basierend auf dem Modell der PGE-Produktgenerationsentwicklung Wissen über die Auswirkungen von Referenzen und Variationen auf Entwicklungszielgrößen situationsgerecht und intuitiv zugänglich macht.

\section{Stand der Forschung}

\section{Einflussfaktoren auf Entwicklungszielgrößen im Modell der PGE}

Das Modell der PGE nach Albers beruht auf der Beobachtung, dass Produkte nicht von Grund auf neu entwickelt werden, sondern immer auf Referenzen und vorangegangenen Entwicklungsgenerationen basieren. Grundlage des Modells der PGE sind zwei zentrale Hypothesen. (Albers, Bursac \& Wintergerst, 2015; Albers, Rapp, Spadinger, Richter, Birk, Marthaler et al., 2019; Albers, Rapp, Fahl, Hirschter, Revfi, Schulz et al., 2020):

Jedes Produkt wird auf der Basis eines Referenzsystems $R_{n}$ entwickelt. Referenzsystemelemente (RSE) stammen aus bestehenden oder bereits geplanten soziotechnischen Systemen und der zugehörigen Dokumentation und dienen als Basis und Ausgangspunkt für die Entwicklung einer neuen Produktgeneration $G_{n}$.

Die Subsysteme eines neuen Produkts werden ausschließlich durch drei Arten von Variationen auf Basis von Referenzsystemelementen entwickelt (siehe Abbildung 1, links): Bei der Übernahmevariation eines Subsystems (ÜV) wird das entsprechende Referenzsystemelement übernommen und bei der Systemintegration gegebenenfalls nur an den Schnittstellen angepasst. Bei der Variation von Attributen (AV) handelt es sich um die Neuentwicklung eines Subsystems unter Beibehaltung des Lösungsprinzips des Referenzsystemelements und Änderung funktionsbestimmender Attribute. Bei der Neuentwicklung durch Prinzipvariation (PV) wird die Funktion des Referenzsystemelements durch ein alternatives Lösungsprinzip erfüllt. 

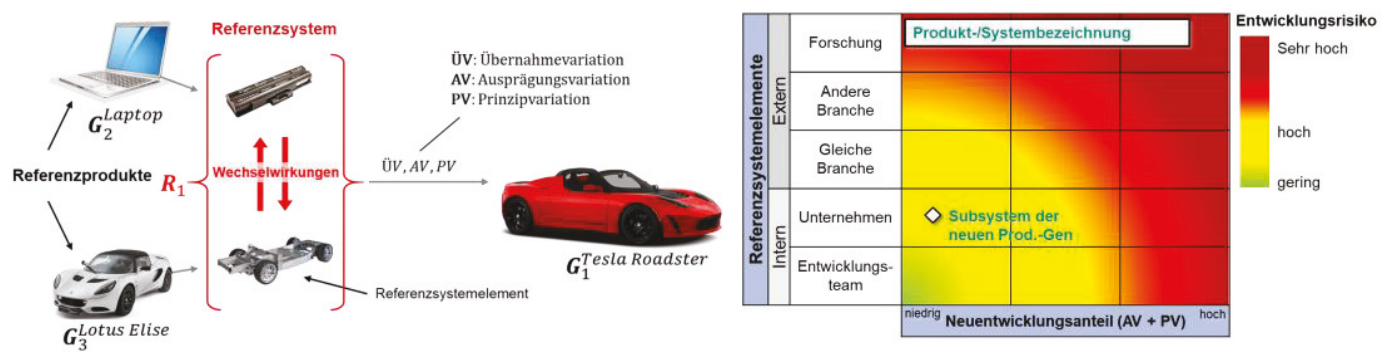

Abbildung 1: Links: Das Referenzsystem im Modell der PGE (Albers, Rapp, Spadinger, Richter, Birk, Marthaler et al., 2019). Rechts: PGE-Risikoportfolio (Albers, Rapp, Birk \& Bursac, 2017).

Das Verhältnis der Anzahl an durch eine bestimmte Variationsart entwickelten Subsystemen zur Gesamtanzahl an betrachteten Subsystemen der neuen Produktgeneration ergibt den Variationsanteil einer Variationsart. Die Anteile von AV und PV zusammengenommen bilden dabei den Neuentwicklungsanteil.

Empirische Untersuchungen in diversen Fallbeispielen haben gezeigt, dass die Charakteristika der verwendeten Referenzsystemelemente und die Variationsart, mit der neue Subsysteme entwickelt werden, Einflussfaktoren auf Entwicklungszielgrößen wie Kosten, Risiko, Innovationspotenzial und durchzuführende Entwicklungsaktivitäten darstellen (z.Bsp. Albers, Bursac \& Rapp 2017). Das PGE-Risikoportfolio visualisiert die Auswirkungen der Herkunft von RSE und des Neuentwicklungsanteils auf das Risiko des betrachteten Subsystems der $\mathrm{G}_{n}$ (siehe Abbildung 1, links).

Als besonders relevant stellen sich die Auswirkungen der Variationsart, der Herkunft des RSE, der Komplexität des RSE und des Reifegrads des RSE auf die Entwicklungszielgrößen Kosten, Risiko, Innovationspotenzial und die durchzuführenden Entwicklungsaktivitäten heraus. Die Überführung dieser Zusammenhänge in ein Entity-Relationship Datenmodell nach Chen (Chen, 1976) macht das Wissen für die computerbasierte Verarbeitung und Visualisierung zugänglich (Pfaff, Rapp \& Albers, 2021, in Druck).

\section{Entscheidungsfindung im Produktentwicklungsprozess}

Entscheidungsfindung im Produktentwicklungsprozess ist häufig auf das Entscheiden zwischen mehreren Lösungsalternativen zurückzuführen (Ehrlenspiel \& Meerkamm, 2017, S. 634). Die dafür vorzunehmende Bewertung einzelner Lösungsalternativen basiert auf aus den Anforderungen an das Produkt hergeleiteten Bewertungskriterien, die mit einem Gewicht multipliziert in das Gesamtergebnis eingehen (Breiing \& Knosala, 1997). 


\section{Visualisierung zur Unterstützung von Entscheidungsfindung}

Nach CARD et al. ist die Visualisierung von Informationen im Kontext computergenerierter Visualisierungen die "interaktive, visuelle Darstellungen von abstrakten Daten zur Verstärkung der Kognition" (Card, Mackinlay \& Shneiderman, 1999, S. 6). Der Zweck von Visualisierungen ist es, durch einen tieferen Einblick Erkenntnisgewinn, Entscheidungsfindung und Erklärung zu ermöglichen. Visualisierungen profitieren von hochwertiger Darstellung und interaktiver Manipulation dieser Darstellungen durch den Anwender (Card, Mackinlay \& Shneiderman, 1999, S. 6).

Virtual-Reality (VR) Umgebungen bieten das Potenzial, durch betrachterabhängige immersive Präsentation und eine besonders natürliche und intuitive Interaktion den Umgang mit komplexen Informations- und Wissensräumen zu vereinfachen (Dörner, Broll, Grimm \& Jung, 2019, S. 15f). Im Realitäts-Virtualitäts-Kontinuum nach Milgram et al. stellt VR das Extrem einer vollständig virtuellen Umgebung dar (Milgram, Takemura, Utsumi \& Kishino, 1995). In der Produktentwicklung wird Virtual Reality unter anderem für virtuelle Validierungsumgebungen eingesetzt (Chen \& Fragomeni, 2019).

\section{Forschungsziel und Forschungsmethode}

Das übergeordnete Ziel dieses Beitrags ist die Unterstützung der Entwickelnden im Entscheidungsprozess durch das Zugänglichmachen von Wissen über die Auswirkungen von Variationen und Referenzen durch Visualisierung. Die theoretische Grundlage für die Unterstützung bildet die formalisierte Beschreibung der Einflüsse der Variationsart und der Charakteristika der Referenzsystemelemente auf Entwicklungszielgrößen im Modell der PGE. Die zu entwickelnde Unterstützung soll die Kriterien Anwendbarkeit, Benutzerfreundlichkeit und Nützlichkeit nach Blessing und Chakrabarti berücksichtigen (Blessing \& Chakrabarti, 2009). Hieraus ergeben sich folgende Forschungsfragen für diese Arbeit:

- Wie kann eine Visualisierung gestaltet werden, die dem Entwickelnden die Auswirkungen von Variationsarten und den Charakteristika der Referenzsystemelemente als Einflussfaktoren auf Entwicklungszielgrößen der Gn zur Entscheidungsunterstützung zugänglich macht?

- Inwieweit eignen sich die entwickelten Visualisierungsansätze hinsichtlich Anwendbarkeit, Benutzerfreundlichkeit und Nützlichkeit für die Unterstützung von Entwickelnden bei der Entscheidungsfindung?

Zuerst wird die grundsätzliche Anwendbarkeit der Zusammenhänge im Entscheidungsprozess und die Nützlichkeit bei der Unterstützung der Entscheidungsfindung 
untersucht. Dazu werden die Zusammenhänge zwischen Einflussfaktoren und Entwicklungszielgrößen im Modell der PGE diagrammbasiert visualisiert und in einem Studierendenworkshop bei der Bewertung von Konzepten angewandt. Die Ergebnisse aus dieser ersten Anwendung fließen in die Entwicklung eines VR-Visualisierungsansatzes mit ein.

\section{Vorstudie in einem Studierendenprojekt zur Validierung der Nützlichkeit des Ansatzes}

Die Studierenden der HECTOR School of Engineering and Management des KIT durchlaufen jedes Jahr ein Produktentwicklungsprojekt, das mithilfe von Methoden und Prozessen der PGE bearbeitet wird. Das Projekt im Wintersemester 2019/20 und Sommersemester 2020 war die Entwicklung der dritten Produktgeneration eines autonomen Bierlieferroboters "Pluto ${ }_{3}{ }^{\prime}$. Zur Unterstützung der Studierenden in der Konzeptphase wurde ein 90-minütiger Workshop zur Generierung und Bewertung von Lösungskonzepten abgehalten. Am Workshop nahmen insgesamt sieben Studierende teil. Diese wurden in drei Gruppen (3-2-2) entsprechend ihrer Verantwortlichkeiten für Antriebsstrang, Elektronik und Steuerung und Mensch-Maschine-Schnittstelle eingeteilt.

\section{Erster Durchgang ohne Visualisierung}

Basierend auf schon vorhandenen Verbesserungsideen für $\mathrm{Pluto}_{3}$ wurden auf vorbereiteten Templates die Lösungskonzepte expliziert. Dabei waren auf der linken Seite die Referenzsystemelemente und auf der rechten Seite die Subsysteme des neuen Konzepts darzustellen und die Variationsbeziehungen zu kennzeichnen (siehe Abbildung 2, links). Mit den ausgefüllten Templates als Bewertungsgrundlage wurde eine erste Bewertungsrunde in den Teams durchgeführt und die Konzepte nach Kosten, Risiko, Innovationspotenzial und Funktionsqualität bewertet (siehe Abbildung 2, rechts).

\section{Zweiter Durchgang mit Visualisierung}

Für den zweiten Durchgang wurde den Teams eine Visualisierung zur Verfügung gestellt, welche je nach Ausprägung der Variationsart und der Charakteristika von RSE (Herkunft, Komplexität, Reifegrad) die Auswirkungen auf die in Bewertungskriterien überführten Entwicklungszielgrößen Kosten, Risiko und Innovationspotenzial darstellt (Abbildung 3). 
Im zweiten Bewertungsdurchgang war es Aufgabe der Studierenden, die Bewertung aus Durchgang eins, falls nötig, aufgrund der zusätzlichen Informationen anzupassen und entsprechend zu kommentieren (siehe Abbildung 4).
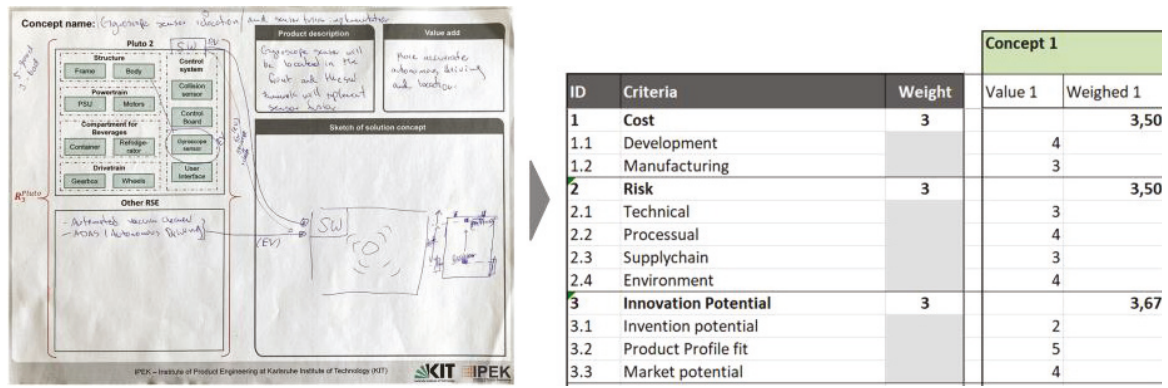

Abbildung 2: Links: Ausgefülltes Template der Gruppe 2 für ein neues Sensorkonzept basierend auf Referenzsystemelementen aus der Vorgängergeneration, Saugrobotern und autonomen Fahrzeugen.

Rechts: Auszug des Bewertungsschemas im ersten Durchgang von Gruppe 1.

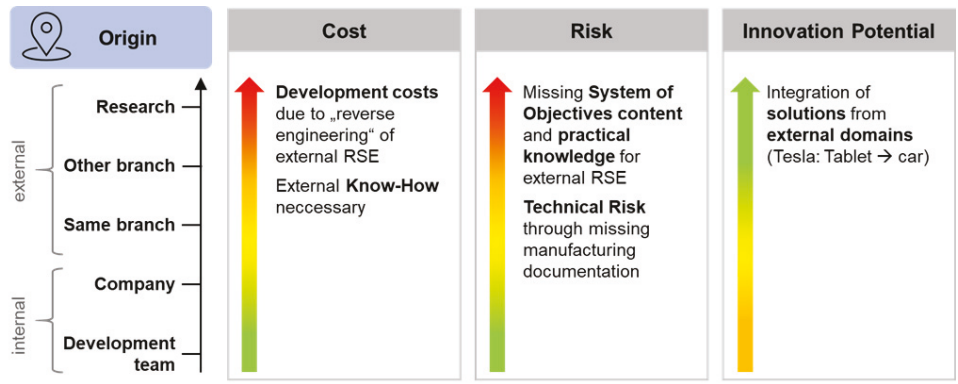

Abbildung 3: Visualisierung für die Auswirkungen am Beispiel der Herkunft von RSE

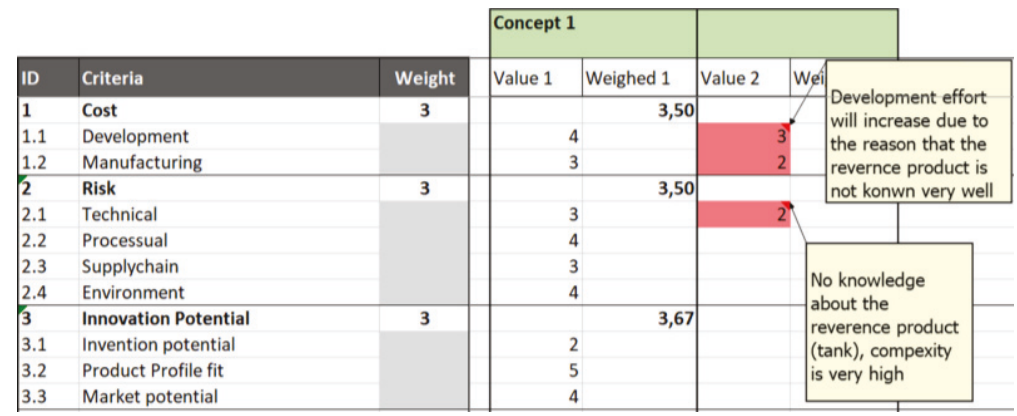

Abbildung 4: Bewertung von Gruppe 1 nach dem zweiten Durchgang 
Team 1 - Antriebsstrang: Die Bewertung des technischen Risikos und der Entwicklungs- und Herstellungskosten für einen Kettenantrieb wurden aufgrund der externen Herkunft und hohen Komplexität des verwendeten Referenzsystemelements (Kettenantrieb eines Panzers) ins Negative angepasst.

Team 2 - Steuerung und Regelung: Hier wurden keine Bewertungen angepasst, jedoch konnte die bisherige Bewertung durch neue Argumente untermauert werden. So wurde die Einschätzung von hohem technischem Risiko bei der Entwicklung von Algorithmen für autonomes Fahren durch die externe Herkunft von Referenzsystemelementen bestätigt.

Team 3 - Mensch-Maschine Schnittstelle: Sowohl Risiko als auch Innovationspotenzial des Konzepts wurden aufgrund der Variationsart und der Herkunft des Referenzsystemelements deutlich schlechter bewertet. Die Negativanpassung des Innovationspotenzials wurde aufgrund von hohen Übernahmeanteilen aus anderen Referenzsystemelementen vorgenommen.

\section{Diskussion des Visualisierungsansatzes der Vorstudie}

In der Vorstudie konnte beobachtet werden, dass das Grundkonzept der Unterstützung von Entwickelnden durch die Bereitstellung von Wissen über die Auswirkungen von Referenzsystemelementen und Variationen auf Bewertungskriterien der $G_{n}$ eine bessere Bewertungsgrundlage liefert. Durch Hinzuziehen der Visualisierung konnten die Studierenden in ihrem spezifischen Entwicklungskontext Eigenschaften von Referenzsystemelementen als Einflussfaktoren identifizieren und über deren Ausprägung auf Auswirkungen auf die Kriterien schließen und diese in der Bewertung berücksichtigen. Die Anwendbarkeit des Unterstützungskonzepts kann daher positiv bewertet werden.

Die Benutzerfreundlichkeit der Visualisierung wird eher negativ bewertet. Die Darstellung ist für die verwendete Menge an Information zu kompliziert und zu komplex. Ein weiterer Kritikpunkt in Bezug auf die Benutzerfreundlichkeit war die Anwendung auf Gesamtsystemebene. Hier würde eine Betrachtung der Auswirkungen auf Subsystemebene favorisiert werden.

Die Nützlichkeit wurde von den Teilnehmenden als positiv bewertet. Durch den Einbezug der Zusatzinformationen in den Bewertungsprozess konnte der Reifegrad der Bewertungsgrundlage und damit die Belastbarkeit der Bewertung erhöht werden. 


\section{Entwicklung einer VR-Umgebung für die Visualisierung der Zusammenhänge zur Bewertungs- und Entscheidungsunterstützung}

Aus den Ergebnissen der Vorstudie ergibt sich der Bedarf für eine benutzerfreundliche, interaktive Visualisierungsumgebung, die das Wissen über die Auswirkungen von Variationsarten und Charakteristika von Referenzsystemelementen auf Zielgrößen der $G_{n}$ auf Subsystemebene übersichtlich und situationsgerecht darstellt. Mit einem VRVisualisierungsansatz sollen die Möglichkeiten der VR-Technologie genutzt werden, um Entwickelnden interaktiv Wissen zugänglich zu machen.

\section{Grundlegende Architektur der VR-Umgebung}

Auf Basis des Entity-Relationship Datenmodells wird das Back-End der automatisierten Auswertung in der 3D-Engine Unity umgesetzt. In der produktunabhängigen Wissensbasis sind die Auswirkungen von Variationsart, der Herkunft der RSE, der Komplexität der RSE und des Reifegrads der RSE auf die Entwicklungszielgrößen Kosten, Risiko, Innovationspotenzial und die durchzuführenden Entwicklungsaktivitäten gespeichert. (Pfaff, Rapp \& Albers, 2021, in Druck).

Im Unity-Editor werden die 3D-Modelle der RSE und der $G_{n}$ importiert. Die Ausprägungen der Charakteristika der RSE werden ebenso wie die Variationsbeziehungen der RSE zu den Subsystemen der $G_{n}$ mithilfe vordefinierter Eingabemasken angelegt. Diese, vom Nutzer einzugebenden Informationen, stellen die produktbezogene Datenbasis dar.

\section{Funktionsweise der VR-Umgebung anhand eines Beispielszenarios}

Für die erstmalige Implementierung der VR-Umgebung wurde ein Mock-Up basierend auf einer beispielhaften Entwicklungssituation erstellt: "Ein Fahrradhersteller möchte einen E-Scooter $\left(G_{n}\right)$ entwickeln, der im Vergleich zu Konkurrenzprodukten einen höheren Komfort und bessere Fahreigenschaften bieten soll. Dazu werden RSE aus anderen Entwicklungsteams (Herkunft: intern, Unternehmen) und Wettbewerbern der Fahrradindustrie (Herkunft: extern, gleiche Branche) als Grundlage für die Entwicklung der Subsysteme Fahrwerk, Bremse und Antriebskomponenten herangezogen." Im Folgenden wird das Vorgehen beispielhaft anhand des Subsystems Radnabenmotor der neuen Produktgeneration und dem zugehörigen Referenzsystemelement Mittelmotor erläutert.

Im E-Scooter-Szenario wird angenommen, dass für den neu zu entwickelnden Radnabenmotor als RSE ein Fahrrad-Mittelmotor eines Zulieferers zur Verfügung steht. Die Ausprägung des Einflussfaktors Herkunft ist daher extern, gleiche Branche, was aufgrund fehlender Zielsysteminhalte und daraus folgendem zusätzlichem Validierungsaufwand tendenziell negative Auswirkungen auf Kosten und Risiko hat (Albers, Rapp, 
Birk \& Bursac, 2017). Die Komplexität des RSE Mittelmotor wird hoch eingestuft. Hohe Systemkomplexität führt im Allgemeinen zu steigenden Entwicklungskosten, da auch die Komplexität der Entwicklungsaufgabe steigt (Hubka, 1984). Mit zunehmender Komplexität steigt auch technische Risiko im Produktentwicklungsprozess (Wißler, 2006). Das RSE Mittelmotor liegt als Serienprodukt vor mit hohem Reifegrad vor. Ein hoher Produktreifegrad bedeutet einen hohen Grad der Erfüllung der Forderungen an das Produkt und damit abgeschlossene Validierungs- und Verifizierungsaktivitäten (Weinzierl, 2006, 20f). Dies hat tendenziell positive Auswirkungen auf Kosten und Risiko im Projekt. Gegenüber dem RSE Mittelmotor stellt der Radnabenmotor eine Neuentwicklung durch Prinzipvariation dar. Ein hoher Neuentwicklungsanteil steigert den Entwicklungsaufwand und führt häufig auch im Produktionssystem zu Änderungen, was tendenziell sehr negativen Einfluss auf Kosten und Risiko im Projekt hat (Albers, Rapp, Birk \& Bursac, 2017; Albers, Bursac \& Rapp, 2017). Ein höherer Neuentwicklungsanteil durch Prinzipvariation ermöglicht unter anderem die Differenzierung gegenüber Wettbewerbern und die Realisierung von Begeisterungsfaktoren, was das Innovationspotenzial tendenziell steigert (Albers, Bursac, Urbanec, Lüdcke \& Rachenkova, 2014; Bailom, Hinterhuber, Matzler \& Sauerwein, 1996; Heismann \& Maul, 2012). Prinzipvariationen erfordern vermehrt die Aktivitäten Prinzip und Gestalt Modellieren und Validieren und Verifizieren (Albers, Bursac \& Rapp, 2017).

Tabelle 1 zeigt die Auswirkungen der Einflussfaktoren auf die Entwicklungszielgrößen für den Radnabenmotor im fiktiven Entwicklungsszenario nochmals zusammengefasst.

Tabelle 1: Auswirkung der Ausprägung der Einflussfaktoren auf die Entwicklungszielgrößen

\begin{tabular}{|c|c|c|c|c|}
\hline $\begin{array}{l}\text { Einflussfak- } \\
\text { tor }\end{array}$ & Ausprägung & Kosten & Risiko & $\begin{array}{c}\text { Innovations- } \\
\text { potenzial }\end{array}$ \\
\hline Herkunft & Extern, gleiche Branche & 2 & 2 & 3 \\
\hline Komplexität & hoch & 2 & 2 & 3 \\
\hline Reifegrad & hoch (Serie) & 4 & 4 & 3 \\
\hline Variationsart & Prinzpvariation & 1 & 1 & 5 \\
\hline \multicolumn{2}{|c|}{ Subsystem der $\mathrm{G}_{\mathrm{n}}$ : Radnabenmotor } & 2,25 & 2,25 & 3,5 \\
\hline $\begin{array}{l}\text { Legende für } \\
\text { die Auswir- }\end{array}$ & 1: sehr negativ & 3: neutral & 4: positiv & 5: sehr positiv \\
\hline
\end{tabular}




\section{Interaktion des Nutzers mit der VR-Umgebung}

Der Nutzer kann die Ergebnisse für die Entwicklungszielgrößen der aktuellen Produktgeneration auf Subsystemebene durch Interaktion mit den Modellen in der Visualisierungsumgebung abrufen und sich bei Bedarf Detailinformation anzeigen lassen (siehe Abbildung 5).

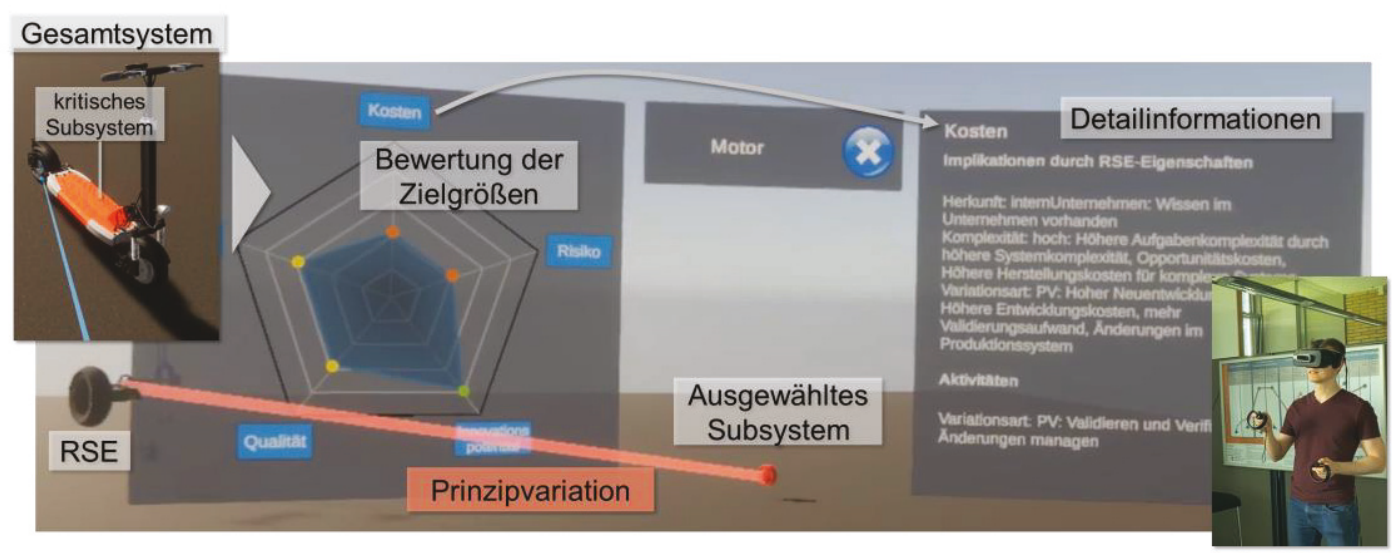

Abbildung 5: Visualisierung der Auswirkungen der Einflussfaktoren auf die Entwicklungszielgrößen im Spinnendiagramm (links).

Das Heranzoomen auf interessierende Subsysteme geschieht in der VR-Umgebung (Abbildung 5) über die Bewegung hin zum Objekt. Dadurch kann der Nutzer sich frei und intuitiv am oder im System bewegen. Durch die Laserpointer wählt der User die Subsysteme aus, zu denen er detailliertere Informationen möchte. Ebenso ist die Interaktion mit den Modellen des Referenzsystems und der $G_{n}$ über Griff-Gesten möglich. Ein roter Strahl vom untersuchten Subsystem Motor (Radnabenmotor) zum RSE Mittelmotor zeigt die Prinzipvariation an. Die Bewertungsergebnisse der Bewertungskriterien (vgl. Tabelle 1) werden in einem Spinnendiagramm auf dem linken Panel dargestellt. Neben Kosten, Risiko und Innovationspotenzial sind in Abbildung 5 noch die Zielgrößen Qualität und Nachhaltigkeit aufgeführt, auf die bisher noch keine direkten Einflüsse identifiziert werden konnten. Das rechte Panel zeigt detaillierte Informationen zur ausgewählten Entwicklungszielgröße. Dadurch wird nachvollziehbar, von welchen Einflussgrößen die Zielgröße beeinflusst wird und welche Entwicklungsaktivitäten notwendig sein können. 


\section{Diskussion der VR-Visualisierungsansatzes}

Die VR-Umgebung wurde initial in einer Diskussion mit Forschenden im Bereich Entwicklungsmethodik, die an der Universität und in verschiedenen Unternehmen tätig sind, validiert. Dort wurden die Anwendbarkeit und Benutzerfreundlichkeit der Anwendung positiv bewertet.

Vergleicht man den VR-Ansatz mit einer klassischen Bildschirm-basierten 3D Visualisierung, ergeben sich neben den intuitiven Bedienmöglichkeiten besonders Vorteile durch den hohen Immersionsgrad. Durch die immersive Interaktion mit den RSE und $\operatorname{der} G_{n}$ in der VR-Umgebung werden Denk- und Assoziationsprozesse angeregt. Gegenüber Bildschirm-basierter Visualisierung hat die VR-Umgebung Nachteile bei der Lesbarkeit von zweidimensionalen Informationen wie Diagrammen und Text, je nach Blickwinkel zur Darstellungsebene. Für den nutzenstiftenden Einsatz von VR-Umgebungen zur Entscheidungsunterstützung in der Praxis gilt es daher, die Nützlichkeit und Anwendbarkeit unter der Nutzung der Vorteile der Benutzerfreundlichkeit auszubauen. Potenzial liegt hier zum Beispiel darin, dem Nutzer dynamisch zur Laufzeit Input zu den Referenzsystemelementen und den Subsystemen der $G_{n}$ zu ermöglichen und so die Entscheidungsgrundlage weiter zu verbessern.

Die Verkürzung des Modells der Variationsbeziehung auf eine 1:1 Beziehung muss für die praktische Anwendung ebenso wie die vorgenommene Vereinfachung auf eine Subsystemebene als Betrachtungsebene aufgehoben werden. Auch die Wechselwirkungen der RSE untereinander sollen in zukünftigen Ansätzen berücksichtigt werden (vgl. Abbildung 1), um beispielsweise Schnittstellenproblematiken früher aufdecken zu können. Eine weitere Herausforderung für die Anwendbarkeit des VR-Ansatzes speziell für frühe Phasen des Produktentwicklungsprozesses liegt in der Notwendigkeit von 3D-Modellen, die bereits lösungsspezifisch sind. Das Wissen über die Auswirkungen der Einflussfaktoren im Modell der PGE bietet auch für Kreativprozesse Potenzial. Hierzu werden in der VR-Visualisierung auch lösungsoffene Repräsentationen von Subsystemen und Designräumen benötigt. Ansätze für solche VR basierten Kreativitätsumgebungen zeigt beispielsweise Mantwill (2020).

\section{Zusammenfassung und Ausblick}

Mithilfe der entwickelten VR-Visualisierungsumgebung kann das Wissen über die Auswirkungen der Variationsart und der Charakteristika der Referenzsystemelemente als Einflussfaktoren auf Entwicklungszielgrößen im Modell der PGE interaktiv und intuitiv visualisiert werden. In der Vorstudie konnte das Potenzial nachgewiesen werden, dass 
das Wissen über diese Zusammenhänge die Entscheidungsgrundlage in frühen Phasen des Produktentwicklungsprozesses verbessert.

Die Erkenntnisse dieser Arbeit werden verwendet, um die bestehende VR-Umgebung weiterzuentwickeln und das Wissen aus der PGE-Forschung auch in anderen virtuellen Methoden zielführend zu integrieren. Sowohl für analytische Methoden zur Unterstützung der Entscheidungsfindung als auch für Kreativmethoden bietet der Visualisierungsansatz Potenzial.

\section{Literaturverzeichnis}

Albers, A., Bursac, N., Urbanec, J., Lüdcke, R. \& Rachenkova, G. (2014). Knowledge Management in Product Generation Development - an empirical study. In D. Krause (Hrsg.), 25 hi Symposium Design for X (DFX) 2014 (pp. 13-24). Berlin: TuTech Verlag.

Albers, A., Rapp, S., Birk, C. \& Bursac, N. (2017). Die Frühe Phase der PGE - Produktgenerationsentwicklung. In H. Binz, B. Bertsche, W. Bauer, D. Spath \& D. Roth (Hrsg.), 4. Stuttgarter Symposium für Produktentwicklung SSP 2017 (Beiträge zum Stuttgarter Symposium für Produktentwicklung, S. 345-354). Fraunhofer, Stuttgart.

Albers, A., Rapp, S., Fahl, J., Hirschter, T., Revfi, S., Schulz, M. et al. (2020). Proposing a generalized Desdription of Variations in different types of Systems by the Model of PGE - Product Generation Engineering. In: Proceedings of the Design Society: DESIGN Conference, 1, 2235-2244.

Albers, A., Bursac, N. \& Rapp, S. (2017). PGE - Produktgenerationsentwicklung am Beispiel des Zweimassenschwungrads. Forschung im Ingenieurwesen, 81(1), 13-31.

Albers, A., Bursac, N. \& Wintergerst, E. (2015). Product Generation Development - Importance and Challenges from a Design Research Perspective. In N. E. Mastorakis (Hrsg.), International Conference on Theoretical Mechanics and Applied Mechanics (Recent advances in mechanical engineering series, vol. 13, pp. 16-21).

Albers, A., Rapp, S., Spadinger, M., Richter, T., Birk, C., Marthaler, F. et al. (2019). The Reference System in the Model of PGE: Proposing a Generalized Description of Reference Products and their Interrelations. In P. B. Schaub \& M. Kleinsmann (Hrsg.), Proceedings of the 22nd International Conference on Engineering Design (ICED19) (Proceedings of the Design Society: International Conference on Engineering Design, Bd. 94, vol. 1, pp. 1693-1702).

Bailom, F., Hinterhuber, H. H., Matzler, K. \& Sauerwein, E. (1996). Das Kano-Modell der Kundenzufriedenheit. Marketing ZFP, 18(2), 117-126.

Blessing, L. T. M. \& Chakrabarti, A. (2009). DRM, a design research methodology. Dordrecht: Springer.

Breiing, A. \& Knosala, R. (1997). Bewerten technischer Systeme. Theoretische und methodische Grundlagen bewertungstechnischer Entscheidungshilfen. Berlin, Heidelberg: Springer Berlin Heidelberg. https://doi.org/10.1007/978-3-642-59229-

Card, S. K., Mackinlay, J. D. \& Shneiderman, B. (Hrsg.). (1999). Readings in information visualization. Using vision to think (The Morgan Kaufmann series in interactive technologies). San Francisco, USA: Morgan Kaufmann.

Chen, J. Y.C. \& Fragomeni, G. (2019). Virtual, Augmented and Mixed Reality. Applications and Case Studies (Bd. 11575). Cham: Springer International Publishing. 
Chen, P. P.-S. (1976). The entity-relationship model---toward a unified view of data. ACM Transactions on Database Systems, 1(1), 9-36. https://doi.org/10.1145/320434.320440

Cross, N. (2008). Engineering design methods. Strategies for product design (4. ed.). Chichester: Wiley.

Dörner, R., Broll, W., Grimm, P. \& Jung, B. (2019). Virtual und Augmented Reality (VR/AR). Berlin, Heidelberg: Springer Berlin Heidelberg.

Ehrlenspiel, K. \& Meerkamm, H. (2017). Integrierte Produktentwicklung. Denkabläufe, Methodeneinsatz, Zusammenarbeit (6. Aufl.). München: Carl Hanser Verlag GmbH \& Co. KG.

Heismann, R. \& Maul, L. (2012). Mit systematischem Innovationsmanagement zum Erfolg. In S. Ili \& A. Albers (Hrsg.), Innovation Excellence. Wie Unternehmen ihre Innovationsfähigkeit systematisch steigern (1. Aufl., S. 39-61). Düsseldorf: Symposion Publishing.

Hubka, V. (1984). Theorie Technischer Systeme. Grundlagen einer wissenschaftlichen Konstruktionslehre (Hochschultext, Zweite, völlig neu bearbeitete und erweiterte Auflage). Berlin, Heidelberg, s.l.: Springer Berlin Heidelberg. https://doi.org/10.1007/978-3-662-10446-0

Mantwill, Frank (2020): Virtual Reality zur Unterstützung in frühen Phasen der Produktentwicklung. In: WiGeP News, S.8-9.

Milgram, P., Takemura, H., Utsumi, A. \& Kishino, F. (1995). Augmented reality: a class of displays on the reality-virtuality continuum. In H. Das (Hrsg.), Telemanipulator and Telepresence Technologies (SPIE Proceedings, S. 282-292).

Pfaff, F., Rapp, S., Albers, A. (2021). Modelling and visualizing knowledge on the reference system and variations based on the model of PGE - Product Generation Engineering for decision support. In P. B. Schaub \& M. Kleinsmann (Hrsg.), Proceedings of the 23rd International Conference on Engineering Design (ICED21). In Druck.

Weinzierl, J. (2006). Produktreifegrad-Management in unternehmensübergreifenden Entwicklungsnetzwerken. Ein ganzheitlicher Ansatz zur Entscheidungsunterstützung im strategischen Anlaufmanagement (Fabrikorganisation). Zugl.: Dortmund, Univ., Diss., 2006. Dortmund: Verl. Praxiswissen.

Wißler, F. E. (2006). Ein Verfahren zur Bewertung technischer Risiken in der Phase der Entwicklung komplexer Serienprodukte (IPAIAO-Forschung und -Praxis, Bd. 437). Zugl.: Stuttgart, Univ., Diss., 2006. Stuttgart, Heimsheim: Univ; Jost-Jetter.

\section{Kontakt}

Felix Pfaff, M. Sc.

Simon Rapp, M. Sc.

Univ.-Prof. Dr.-Ing. Dr. h. c. Albert Albers

KIT - Karlsruher Institut für Technologie

IPEK - Institut für Produktentwicklung Campus Süd

Kaiserstr. 10

76131 Karlsruhe

http://Www.ipek.kit.edu/index.php 